\title{
Lower Limb Fractures in the Chronic Spinal Cord Injured Patient
}

\author{
R. R. Ingram, FRCS, ${ }^{1}$ R. K. Suman FRCS, $M S,{ }^{2}$ P. A. Freeman FRCS, \\ $\mathbf{M A}^{3}$ \\ ${ }^{1}$ Orthopaedic Registrar, ${ }^{2}$ Orthopaedic Senior Registrar, ${ }^{3}$ Consultant Orthopaedic \\ Surgeon, West of Scotland Regional Spinal Injury Unit, Philipshill Hospital, East \\ Kilbride Road, Clarkston, Glasgow, UK.
}

\begin{abstract}
Summary
Experience in managing 33 lower limb fractures in 25 chronic spinal injured patients is described. An increased awareness among patients and medical staff is required to avoid misdiagnosis. Effort should be made to ensure bone union in satisfactory position, recognising the different requirements of ambulant and non-ambulant patients. 'Soft pillow splints' were found to give the best results in all fractures other than those of the femoral neck. Operative treatment with antibiotic prophylaxis is only indicated to correct important deformity.
\end{abstract}

Key words: Chronic spinal cord injury; Lower limb fractures.

Improvements in the rehabilitation and care of quadriplegic and paraplegic patients are resulting in longer life spans for these people, especially those surviving the first year after injury (Mesard et al., 1978). In addition, the better quality of life allows a far greater range of activity than was previously possible. Combined with factors such as osteoporosis and muscle weakness, lower limb fractures are not uncommon in this population. Incidences of 1 to $6 \%$ have been reported (Comarr, Hutchinson, Bors, 1962; Eichenholz, 1963; Ragnarsson, Sell, 1981). The problems of osteoporotic bones, anaesthetic skin and recurrent bacteraemia, dictate a different approach in the management of fractures in this population. In addition, the propensity for exuberant callus formation in the paralysed limb has been well documented (Comarr, Hutchinson, Bors, 1962; Eichenholz, 1963; Freehafer, Mast, 1965).

This paper reports on the experience of managing lower limb fractures in a population of 526 chronic spinal injury patients over a period of 15 years, from 1971 onwards, at the West of Scotland Regional Spinal Injury Unit, Glasgow.

\section{Clinical material}

The records of 526 patients were examined and 25 patients with 33 lower limb fractures were identified (incidence of $5^{\circ}$ ). The aetiology of the paralyses in 
Table Aetiology of spinal paralysis

\begin{tabular}{lr}
\hline Falls from heights & 12 \\
Road traffic accidents & 4 \\
Mining accidents & 2 \\
Spina bifida & 2 \\
Dermoid cysts & 2 \\
Acute ascending myelitis & 1 \\
Pyogenic spinal abscess & 1 \\
Unknown aetiology & 1 \\
\hline
\end{tabular}

this series are shown in the Table. The vertebral levels of spinal damage were: 4 cervical, 9 thoracic, 6 thoracolumbar and 6 lumbar. There were 6 femoral neck, 5 femoral shaft, 9 supra-condylar, 1 femoral condylar, 1 patellar and 11 tibial fractures in this series. All were closed fractures.

The ages of patients at time of fracture ranged from 13 to 70 years. The time interval between onset of paralysis and the subsequent fracture varied from 1 to 15 years. Ten fractures were sustained from trivial injuries e.g. during transfer from bed to chair or while simply moving in bed. Twelve fractures resulted from falls, which would not usually have caused a fracture in a normal bone. One occurred during Functional Electrical Stimulation, 1 in a road traffic accident and 1 patient was run over by his own invalid car! In 8 fractures, there was no history of injury.

Five patients, including 1 with a patellar fracture, 1 with a lateral tibial plateau fracture, 2 with tibial shaft fractures and the patient with a fractured neck of femur which presented late, were treated as outpatients. In others, hospitalisation ranged from 1 week to 1 year.

\section{Treatment and results}

Twenty nine fractures were treated conservatively and 4 operatively. The methods chosen altered over the 15 years as experience was gained.

\section{Femoral neck fractures}

There were 6 fractured necks of femur. A young man sustained a grade 4 sub-capital fracture while undergoing Functional Electrical Stimulation. The diagnosis was delayed by 1 week. His fracture was treated by Thompson's Hemiarthroplasty to allow him to regain his former mobility. This however was subsequently complicated by a fracture of the proximal shaft of femur a few days later, which was managed by skeletal traction. This healed well, but he had a stiff hip, due mainly to excessive callus formation (Fig. 1).

The other 5 were initially treated by conservative methods, i.e. rest in bed with the fractured leg in abduction. One patient required open reduction and a nail plate to correct a coxa-vara deformity. One fracture was diagnosed 3 to 4 weeks late and remained ununited. This patient retained his mobility with calipers but complained of a 'lax' hip and a short leg. One patient had a large pressure sore over his greater trochanter, prior to the fracture. He required a hip disarticulation. The remaining femoral neck fractures healed in good alignment and achieved their pre-fracture mobility. 


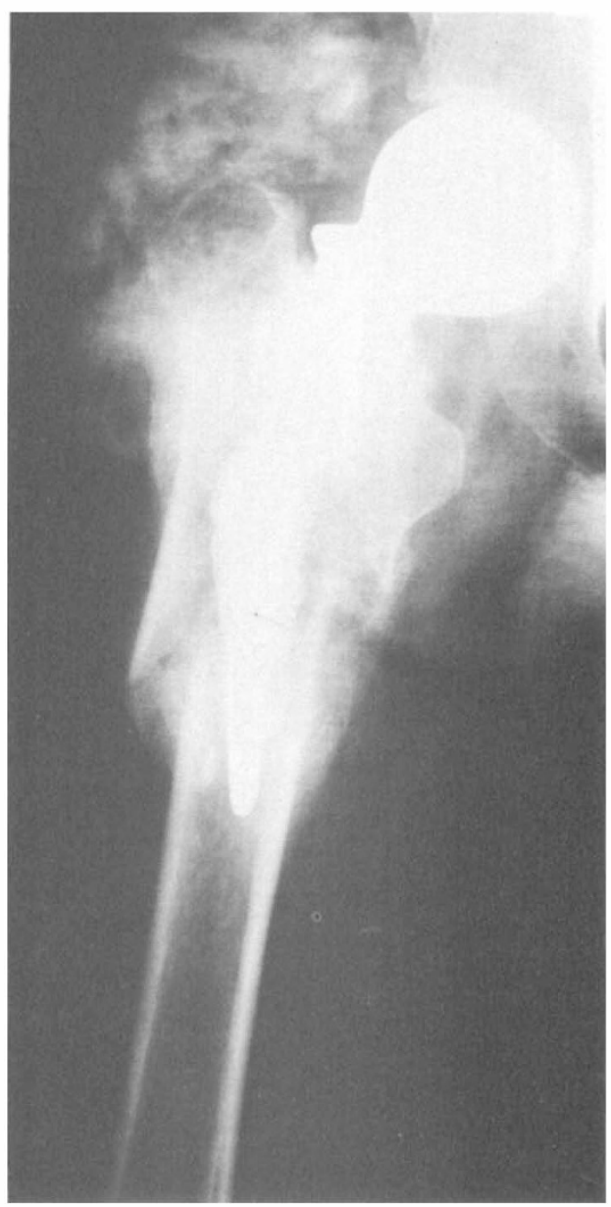

Figure 1 Thompson's Hemi-arthroplasty complicated by a proximal femoral shaft fracture. Exuberant callus formation resulted in a stiff hip.

\section{Femoral shaft fractures}

There were 5 fractured shafts of femur. Two were treated operatively at other hospitals with intra-medullary nails because they developed pressure sores while in Thomas splints. Of these, 1 returned to being mobile with calipers and crutches and the other to his wheelchair existence. The latter however, required a manipulation of his stiff knee under anaesthesia to allow him to sit properly.

Three fractures were treated conservatively. One elderly patient sustained, in addition to the femoral shaft fracture, bilateral supra-condylar fractures of the femur and a proximal tibial fracture. He died of a myocardial infarction shortly after admission to hospital. One fracture, treated in Hamilton-Russell traction, required 3 changes of pin site because of infection and loosening, while another developed a sacral bed sore in traction and required a rotation skin flap.

\section{Supra-condylar fractures}

Nine supra-condylar femoral fractures were treated conservatively by a variety of padded splints. All these patients were wheelchair bound. One, with bilateral 


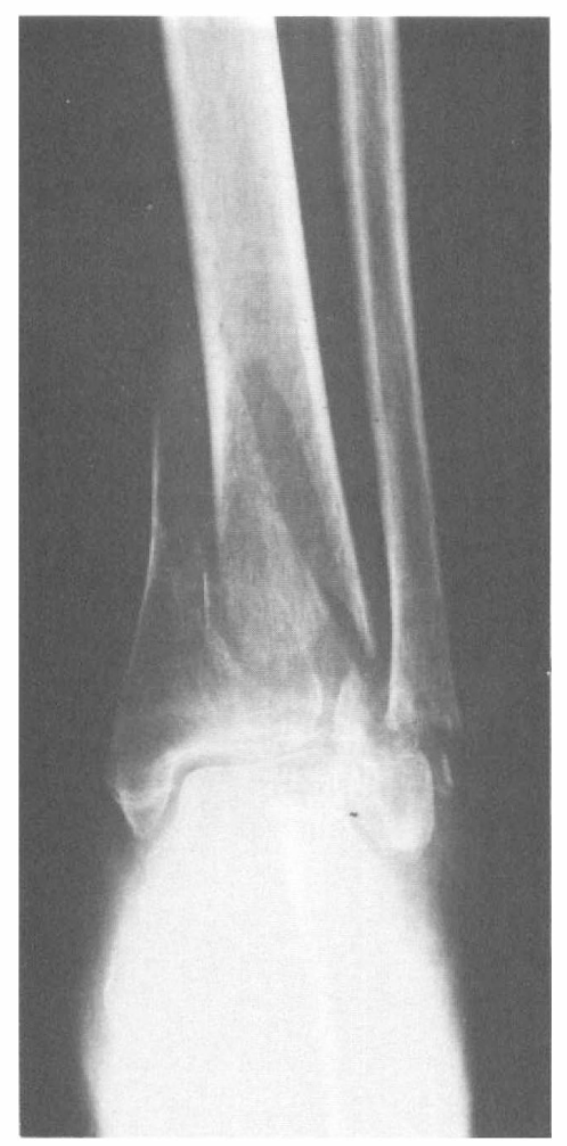

Figure 2A A distal tibial fracture treated in a pillow splint.

fractures as mentioned above, died from a myocardial infarction. The remainder had good results with one exception; he developed a non-union and finally required an above knee amputation. There was 1 undisplaced fracture of a medial femoral condyle which was treated in a simple padded bandage and healed rapidly. One patient with an undisplaced patellar fracture was successfully treated in a plaster cast though she developed a plaster sore over her achilles tendon which took a long time to heal.

\section{Tibial fractures}

Eleven tibial fractures, including 3 of the tibial plateau, were managed conservatively in padded splints (Fig. 2A, Fig. 2B). Four of these patients were mobile with calipers and they returned to this level of activity.

In this series there were no problems with infection in the hemi-anthroplasty, nail plate or intra-medullary nails.

\section{Discussion}

An increased awareness of these fractures, among both patients and medical practitioners, would facilitate early diagnosis. This is important because, for 


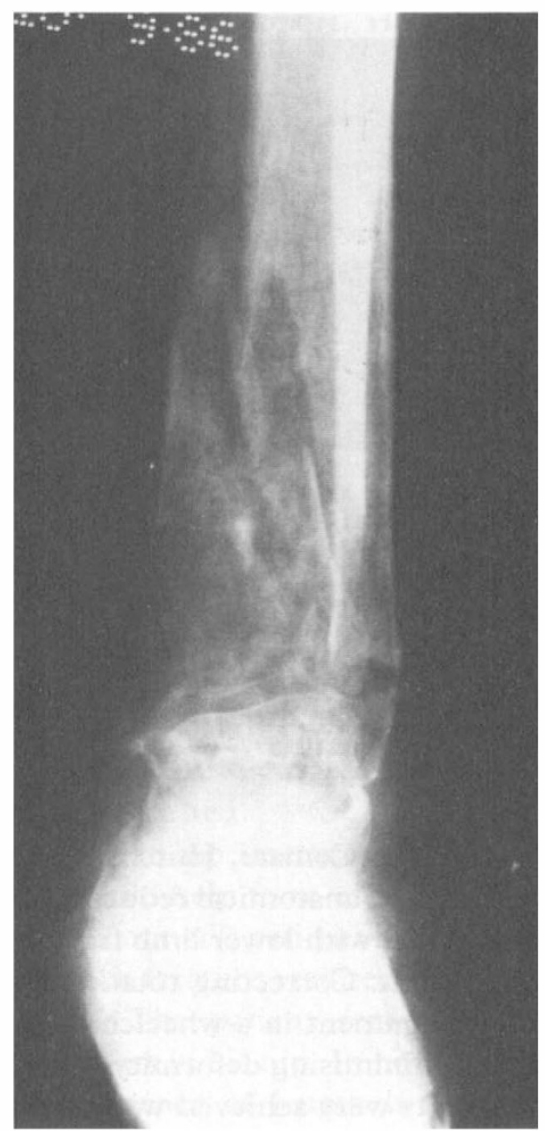

Figure 2B Healed fracture (6 weeks later).

example, splinting may help limit haematoma formation in a limb with poor muscle tone, or prevent later deformity. In many instances, because of diminished or absent pain sensation, there is delay in seeking medical advice. In $12(36 \%)$ fractures in this series, delay in diagnosis ranged from 1 day to 4 weeks. In 7 cases the patients delayed seeking medical advice and in the other 5 the diagnosis was missed or incorrect, e.g. 1 patient presented with a painless swelling and cellulitis of his lower leg and was treated with antibiotics for a week. Later clinical and X-ray examination confirmed a fracture of the tibia. Apparent cellulitis and induration confusing the diagnosis has been noted by Katz (1953).

Management aims include rapid bony union in satisfactory alignment and restoration to pre-fracture of mobility without damage to the skin. In the majority of patients callus formation was rapid, leading to early stability and union. Two non-unions in this series presented late. The non-union of the femoral neck fracture occurred because the patient remained mobile on his painfree limb. One non-ambulant patient with a supra-condylar femoral fracture was treated conservatively. Non-union occurred and subsequently he required an above knee amputation. The incidence of non-union in similar fractures reported 


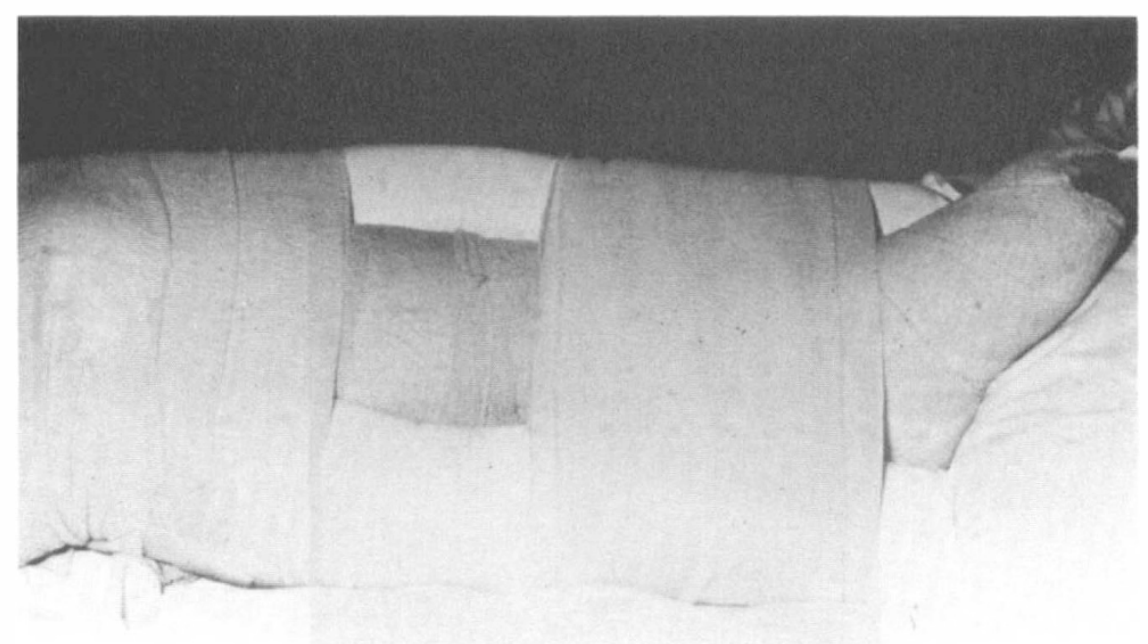

Figure 3 A 'soft pillow splint' on a fractured tibia.

by others ranges from 2 to $10 \%$ (Comarr, Hutchinson, Bors, 1962; Freehafer, Mast, 1965; Nottage, 1981). Exact anatomical reduction of fractures is not necessary. In non-ambulatory patients with lower limb fractures, a degree of shortening or angulation is unimportant. Correcting rotation however, is important in keeping the foot in normal alignment in a wheelchair. In an ambulant patient, maintaining limb length and minimising deformity is more important.

In this series, the best results were achieved with the use of soft, well padded splints. These can be made by aligning one or two pillows along the limb and holding them in position with a crepe bandage. A typical splint of a fractured leg is shown in Figure 3. They are satisfactory in the management of ankle, tibial, femoral shaft and supra-condylar fractures, being economic, effective and safe. When harder materials like Plaster of Paris were used, minor skin breaks were common. Using this method only one supra-condylar femoral fracture failed to unite. Although skin traction was used in two patients without any problem, this would not now be done. The risk of traumatising anaesthetic skin is great (Eichenholz, 1963). Five pin tract infections were encountered during their use in skeletal traction. All of these healed following removal of pins. Skeletal traction would now be avoided where possible.

The hazards of internal fixation and hemi-arthroplasty have been well documented (Eichenholz, 1963; Freehafer, Mast, 1965), and we agree that these outweigh any potential benefits. Although there were no significant problems in the four patients in this series, we do not recommend this as a standard method of treatment. Under certain circumstances however, in an ambulant patient, when conservative methods will not control deformity, e.g. muscle spasm, internal fixation may be necessary. This must be carried out under proper antibiotic cover.

Patients need not be bed-bound during treatment and were often mobile in their wheelchairs. The limbs were supported when necessary on a leg extension of the wheelchair. Hospitalisation should be kept to a minimum, this being 
possible, with adequate community support. However some of these patients may become unwell for a few days immediately after the fracture, with apparent general malaise, cellulitis, fever and rigors, requiring rest in bed. This phenomenon has been noted previously (Freehafer, Hazel, Becker, 1981). The cause for it is not entirely clear, but may be related to fat embolism or the recurrent bacteraemias to which these patients are susceptible.

The use of external fixators on femoral shaft fractures has been reported in a small number of patients with chronic spinal cord damage (Baird et al., 1986). We do not have any experience of this. Their initial results were favourable, but the underlying problem of soft bone resulting in early loosening of pins and pin tract infection is a real one. In the long term this method may not confer any significant advantage over simple splintage.

Fractures around the hip joint are a different problem. Although simple splintage cannot be applied as in other lower limb fractures in this population, the same general rules of fracture management apply. In ambulant patients, rest in bed with the leg supported by pillows in abduction, may suffice. Rapid callus formation in 2 such patients in this series allowed them to be safely mobile in wheelchairs within 6 weeks and walking with their calipers within 10 weeks with good bone union. If the fracture alignment is unacceptable, internal fixation or hemi-arthroplasty may be indicated.

Nottage (1981) treated similar hip fractures aggressively by open reduction and fixation, thinking this would help to re-establish sitting balance. His study did not substantiate this and 2 out of 7 patients had serious complications.

In our series, 1 femoral neck fracture resulted in non-union because of delay in diagnosis and treatment. The patient remained ambulant with calipers although he had some discomfort in his hip. In non-ambulant patients, in whom accurate reduction or maintenance of length is less important, treatment is unnecessary. These patients are generally asymptomatic and operative intervention is contra-indicated.

In the future, fracture prevention may become increasingly important. The roles of e.g. Functional Electrical Stimulation and Calcitonin therapy in preventing osteoporosis have yet to be defined.

\section{References}

BAIRD R, KREITENBERG A, Eltorai I 1986 External fixation of femoral shaft fractures in spinal cord injury patients. Paraplegia 24:183-190.

Comarr AE, Hutchinson RH, Bors E 1962 Extremity fractures of patients with spinal cord injuries. American fournal of Surgery $103: 732-739$.

EICHENHOLZ SN 1963 Management of long bone fractures in paraplegic patients. fournal of Bone and foint Surgery 45-A:299-310.

FReEHAFER AA, HAZEL CM, BECKER CL 1981 Lower extremity fractures in patients with spinal cord injury. Paraplegia 19:367-372.

FREEHAFER AA, MAST WA 1965 Lower extremity fractures in patients with spinal-cord injury. Fournal of Bone and Foint Surgery 47-A:683-694.

KATZ J 1953 Spontaneous fractures in paraplegic children. Fournal of Bone and foint Surgery 35-A:220-226.

Mesard L, Carmody A, Mannarino E, et al. 1978 Survival after spinal cord trauma. A life table analysis. Archives of Neurology 35:78-83.

NotTAGE W 1981 A review of long bone fractures in patients with spinal cord injuries. Clinical Orthopaedics 155:65-70.

RAGNARSSON KT, SELL GH 1981 Lower extremity fractures after spinal cord injury: a retrospective study. Archives of Physical Medicine and Rehabilitation 62:418-423. 